\title{
GPS Anomaly Analysis and Pseudorange Accuracy Improvement by Anomalous Satellite Elimination
}

\author{
Yun-Ja Yoo ${ }^{\dagger} \cdot$ Deuk-Jae Cho* $\cdot$ Sang-Hyun Park** \\ †,*,**Korea Ocean Research \& Development Institute, Daejeon, 305-343, Republic of Korea
}

\begin{abstract}
GPS anomaly has increased according to the degradation of satellite performance, and many GPS users could be exposed to any kinds of error-included signals without any previous notice when unscheduled error occurred. RSIM (Reference Station Integrity Monitors) is a typical monitoring method to broadcast PRC (Pseudo Range Correction) for users. However, there were some cases that the receiver detected the anomalous satellite's signal even though it was unhealthy set, consequently it occurred a large range error. Then it is important to monitor the integrity of GPS signal and it is needed to devise the correction method of pseudorange by eliminating error-occurred PRN for notification to GPS users when it is monitored that the anomaly occurred. This paper proposes the basic concept of how to correct the pseudorange. The paper also shows the analysis results of PRN10 GPS anomaly occurred on day 39 in 2007 with corrected results by eliminating anomaly satellite (PRN10). The proposed correction method shows decreased pseudorange error range compared to the case when the anomaly satellite were used.
\end{abstract}

Key words : GPS anomaly, unscheduled error, pseudorange error, integrity monitoring

\section{Introduction}

It has been reported that increasing numbers of anomaly occur due to the performance degradation of GPS satellite which has limited life span [1,2]. There were more than 13 times of unscheduled outages from NANU (Notice Advisory to Navstar User) every year during 2000 2009 on an average [3]. GPS users could be exposed in those error-occurred situation when GPS anomaly started, and the receiver sometimes tracks the anomaly satellite even though it was set unhealthy by GPS control station.

There are four sections, which are GPS satellite, control station, SIG (Signal In Space) and user surroundings, to cause GPS anomaly and the error by GPS satellite is mainly described in this study. The anomalies occurred by GPS satellite have four main cases which are clock failure, navigation message error, ephemeris error and equipment fault. All of these faults can be resulted in pseudorange error to GPS users [4]. Table 1 listed the well known cases of GPS anomaly classified as four main fault types from 2000 to May of 2010 .

IM (Integrity Monitoring) is to detect an anomalous GPS signal, and there are two conventional IM methods of RSIM (Reference Station Integrity Monitors) by DGPS reference station and RAIM (Receiver Autonomous Integrity Monitoring) by receiver only. RSIM is the integrity monitoring method to broadcast PRC (Pseudo Range Correction) or anomalous GPS satellite's condition to give information for users by DGPS reference station. The position of reference station is already known, then the corrected PR compared to the other station can be generated [5]. However, a large range error sometimes occurs because the receiver detect the anomalous GPS satellite even though the reference station broadcast to users not to use an anomalous signal [6].

RAIM is also the integrity monitoring method by using receiver channels when more than 5 satellites are observed. However, it has a complicated calculation process and low reliability because it uses the redundant measurements.

This paper proposes a new concept of IM by eliminating an anomalous satellite and shows the basic concept of how to detect GPS anomaly and remove an anomalous satellite. It also shows the analysis results about the anomaly case of SVN40/PRN10 (Space Vehicle Number 40 / Pseudo Random Noise 10) which has occurred an ephemeris error. It proposes the basic concept of how to correct pseudorange by eliminating the anomaly-occurred satellite, and the corrected results of pseudorange error and receiver clock bias are shown.

\footnotetext{
† Corresponding Author, yyoo@moeri.re.kr, 042)866-3661

*djcho@moeri.re.kr 042)866-3683

**shpark@moeri.re.kr, 042)866-3681
} 
GPS Anomaly Analysis and Pseudorange Accuracy Improvement by Anomalous Satellite Elimination

Table 1 Well known cases of GPS anomaly classified as for main fault types from 2000 to May of 2010

\begin{tabular}{|c|c|c|c|c|}
\hline Year Fault & $\begin{array}{l}\text { Clock } \\
\text { failure }\end{array}$ & $\begin{array}{c}\text { Navigation } \\
\text { message }\end{array}$ & $\begin{array}{c}\text { Ephemeris } \\
\text { error }\end{array}$ & $\begin{array}{c}\text { Equipment } \\
\text { fault }\end{array}$ \\
\hline 2010 & & & & $\begin{array}{l}\text { SVN39/ } \\
\text { PRN09 }\end{array}$ \\
\hline 2009 & & $\begin{array}{l}\text { SVN54/ } \\
\text { PRN18 }\end{array}$ & & $\begin{array}{l}\text { SVN49/ } \\
\text { PRN01 }\end{array}$ \\
\hline 2007 & $\begin{array}{l}\text { SVN37/ } \\
\text { PRN07 }\end{array}$ & $\begin{array}{l}\text { SVN51/ } \\
\text { PRN20 }\end{array}$ & $\begin{array}{l}\text { SVN40,54/ } \\
\text { PRN10,18 }\end{array}$ & \\
\hline 2006 & & & $\begin{array}{l}\text { SVN24/ } \\
\text { PRN24 }\end{array}$ & \\
\hline 2004 & $\begin{array}{l}\text { SVN23/ } \\
\text { PRN23 }\end{array}$ & & & \\
\hline 2003 & $\begin{array}{l}\text { SVN27,35/ } \\
\text { PRN27,05 }\end{array}$ & & & \\
\hline 2002 & & $\begin{array}{l}\text { SVN21/ } \\
\text { PRN21 }\end{array}$ & & \\
\hline 2001 & $\begin{array}{l}\text { SVN22/ } \\
\text { PRN21 }\end{array}$ & & & \\
\hline 2000 & $\begin{array}{l}\text { SVN14,16/ } \\
\text { PRN14,16 }\end{array}$ & $\begin{array}{l}\text { SVN39/ } \\
\text { PRN09 }\end{array}$ & & \\
\hline
\end{tabular}

\section{Basic Concept of Correction Method}

The basic concept of how to detect the anomaly case is presented and the correction method to enhance the pseudorange accuracy is also proposed when it is monitored that there are anomaly satellites.

Fig. 1 shows the basic concept of anomaly satellite's detection method and how to generate the pseudorange correction by eliminating anomaly satellite.

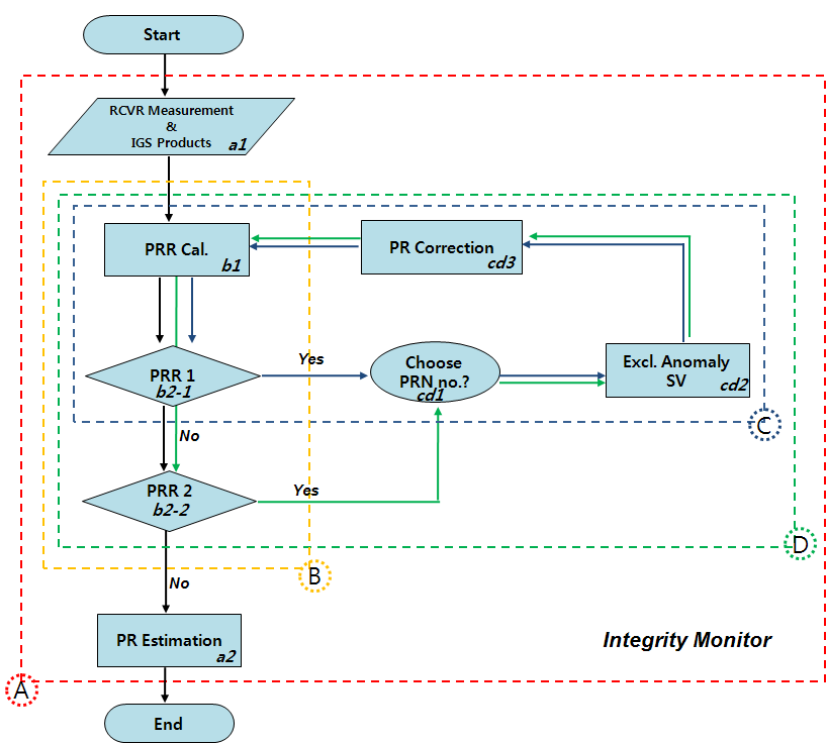

Fig. 1 Basic concept of anomalous PRN elimination and correction method to enhance pseudorange accuracy
Block $\mathrm{A}$ is the integrity monitor section and it is divided into block $\mathrm{B}, \mathrm{C}$ and $\mathrm{D}$. The receiver measurement data such as RINEX (Receiver INdependent EXchange format) navigation data, RINEX obversation data, and IGS (International GNSS Service) products of atmospheric parameters such as the final tropospheric delay and ionospheric delay are received in $a 1$ of block A.

Block B is proceeded when it is not monitored that there is any anomalous signal. PRR (Pseudo Range Rate) is calculated in $b 1$, and the present PRR of $b 2-1$ is compared with IGS data and/or the previous PRR of $b 1$ in block B. If any anomalous signal was not detected in $b 2-1$, the pseudorange in $a 2$ is estimated.

Block $\mathrm{C}$ is proceeded when the anomalous signal is detected in $b 2-1$, and the anomalous PRN number is selected in $c d 1$ of block $\mathrm{C}$. The selected PRN is eliminated in $c d 2$, and the PRC (Pseudo Range Correction) is added in $b 1$. The second PRR comparison is performed in $b 2-2$ and if the second anomalous signal was monitored in $b 2-2$, the second PRN selection is done in $c d 1$, and the selected PRN is eliminated in $c d 2$ of block D. The second PRC is added in $b 1$.

The maximum number of satellite elimination can be explained as follows;

$$
S V_{E N}=S V_{V N}-S V_{M N}
$$

where, $\mathrm{SV}_{\mathrm{EN}}$ is the possible number of satellite elimination, $\mathrm{SV}_{\mathrm{VN}}$ is the number of visible satellites, $\mathrm{SV}_{\mathrm{MN}}$ is the minimum number to get positioning which is more than 4 satellites.

According to IALA (International Association of Marine Aids to Navigation and Lighthouse Authorities) Recommendation R-121, the threshold of pasudorange correction is between $90 \sim 600 \mathrm{~m}$, and the range rate correction is between $0.5 \sim 10 \mathrm{~m} / \mathrm{s}$. Then the threshold of PRR in Fig. 1 can be set less than $10 \mathrm{~m} / \mathrm{s}$ [7].

The measurement equation of pseudorange using $\mathrm{C} / \mathrm{A}$ (Coarse/Acquisition) code and phase measurement equation using carrier phase are defined as follows $[5,8]$.

$$
\begin{aligned}
& \Psi_{R}^{j}=r_{R}^{j}+c\left(B_{R}-B^{j}\right)+I^{j}+T^{j}+M^{j}+\epsilon_{\Psi} \\
& \Phi_{R}^{j}=r_{R}^{j}+c\left(B_{R}-B^{j}\right)-I^{j}+T^{j}+M^{j}+N \lambda+\epsilon_{\Phi}
\end{aligned}
$$

Here, $\Psi$ is the pseudorange measurement $(\mathrm{m}), \Phi$ is the carrier phase measurement $(\mathrm{m}), r$ is the distance between satellite and receiver $(\mathrm{m}), c$ is the light velocity in vacuum 
$(\mathrm{m} / \mathrm{s}), B$ is the clock bias $(\mathrm{s}), I$ is the ionospheric delay $(\mathrm{m})$, $T$ is the tropospheric delay $(\mathrm{m}), M$ is the multipath delay (m), $N$ is the integer of carrier phase ambiguity (cycles), $\lambda$ is the carrier wave length $(\mathrm{m}), \epsilon_{\Psi}$ and $\epsilon_{\Phi}$ are the receiver measurement noise, ${ }^{j}$ is the satellite number, $R$ is the receiver number.

In Equations (2), (3), distance $r$ between satellite and receiver, ionospheric $I$, tropospheric $T$ are as below.

$$
\begin{aligned}
& r_{R}^{j}=\sqrt{\left(X^{j}-x_{R}\right)^{2}+\left(Y^{j}-y_{R}\right)^{2}+\left(Z^{j}-z_{R}\right)^{2}} \\
& \frac{I^{j}}{c}=A_{1}+A_{2} \cos \left(\frac{2 \pi\left(t-A_{3}\right)}{A_{4}}\right) \\
& T^{j}=\frac{2.208}{\sin (E)}\left(e^{\frac{-h_{r}}{6900}}-e^{\frac{-h_{s}}{6900}}\right)
\end{aligned}
$$

where, $X, Y, Z$ are satellite position (m), and $x, y, z$ are receiver position (m), $A_{1}$ is the zenith pass delay at night (sec), $A_{3}$ is the phase when cosine function has maximum value $(\mathrm{m}), A_{2}$ is the amplitude of cosine function in the daytime $(\mathrm{m}), A_{4}$ is the period of cosine function (sec). $A_{2}, A_{4}$ can be obtained from RINEX navigation data. $E$ is the elevation angle of satellite $(\mathrm{deg})$, and $h_{r}, h_{s}$ are the elevation of receiver and satellite $(\mathrm{m})$, respectively. Equations (5), (6) are Klobuchar model, Magnavox model, respectively. However, IGS atmospheric parameters of final tropospheric delay and final ionospheric delay are used instead of Equations (5), (6) for high precision. The satellite position of $X, Y, Z$, the receiver position of $x, y, z$ and clock bias $B$ can be calculated using RINEX navigation data and RINEX observation data. The multipath delay and the measurement noise of receiver are not considered in this paper.

PRE (Pseudo Range Error) is defined as follows;

$$
P R E=P R_{C a l}-P R_{O b s}
$$

where, $P R_{C a l}$ is the calculated pseudorange $(\mathrm{m}), P R_{O b s}$ is the observed pseudorange $(\mathrm{m})$. The measurement equation of pseudorange using Equation (3) is used to analyze PRE in this paper.

\section{PRN10 Anomaly Analysis}

\subsection{Analysis Results}

NANU set PRN10 to be unusable from 18:43 (UTC) on 8th of February (day 39) to 01:12 (UTC) of 9th of February (day 40) in 2007. The error type occurred on PRN10 was a planned ephemeris error. As a result, there was a pseudorange error more than $1.5 \mathrm{~km}$ and other satellites were also affected on pseudorange.

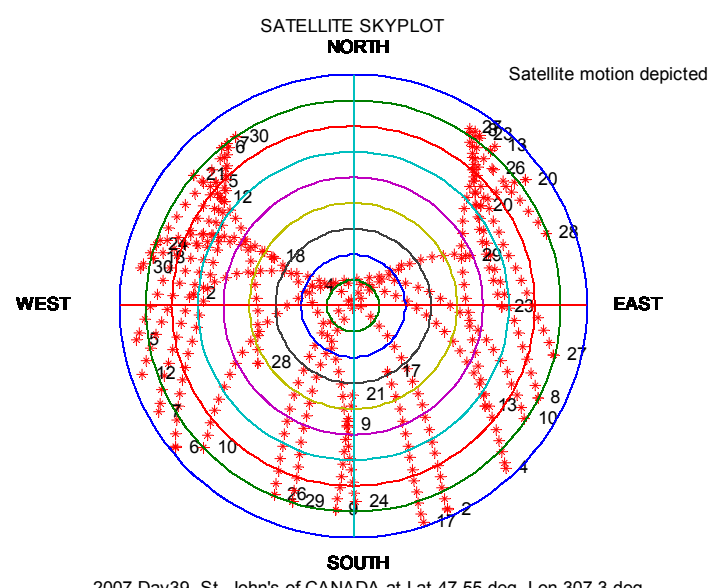

Fig. 2 Visible GPS satellites' motion on 8th of Feb. (day 39) in 2007 from 17:00 23:59 (UTC) at St. John's of Canada
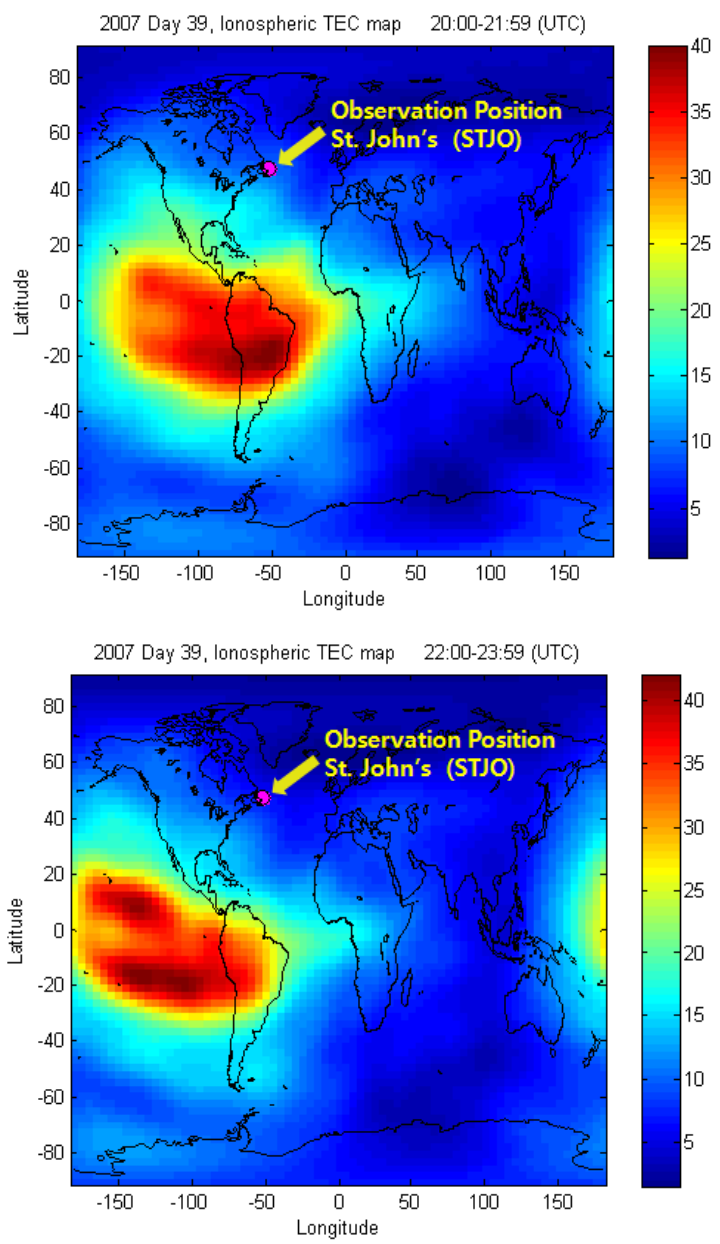

Fig. 3 TEC map during GPS anomaly ocurred on 8th of Feb. (day 39) in 2007 from 20:00 23:59 (UTC) 
GPS Anomaly Analysis and Pseudorange Accuracy Improvement by Anomalous Satellite Elimination

Fig. 2 is the sky plot showing visible satellites' motion at St. John's of Canada (Lat 47.6N, Long 52.7W) from 17:00 23:59 (UTC) on day 39 in 2007.

IGS atmospheric parameters such as final tropospheric zenith path delay and final ionospheric TEC (Total Electron Content) grid were used to estimate pseudorange, and RINEX data was used to calculate pseudorange and pseudorange error.

Fig. 3 shows the TEC map on the day occurred GPS anomaly from 20:00 to 23:59 (UTC) on 8th of February (day 39) in 2007, and it changes within $5 \mathrm{~m} \sim 10 \mathrm{~m}$ near St. John's reference station.

Fig. 4 shows the final tropospheric zenith path delay served by IGS on day 39 in 2007 at St. John's reference station (STJO) of Canada, and it changes within $2.235 \mathrm{~m} \sim$ $2.255 \mathrm{~m}$. The IGS final tropospheric zenith path delay was sampled every 300 seconds so the spline interpolation was used to adjust the data sampling to RINEX data which has 30 seconds sampling period.

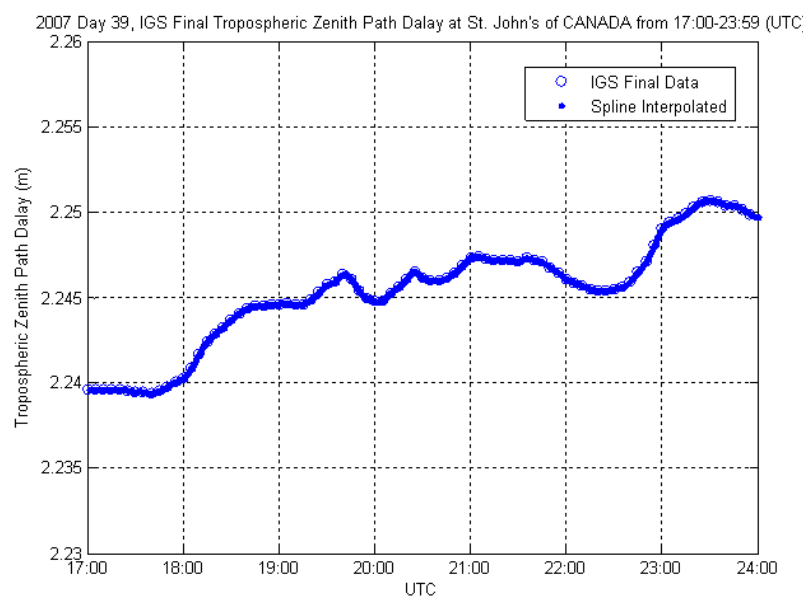

Fig. 4 IGS final tropospheric zenith path delay and spline interpolated results on 8th of Feb. (day 39) in 2007 from 17:00 23:59 (UTC) at St. John's of Canada

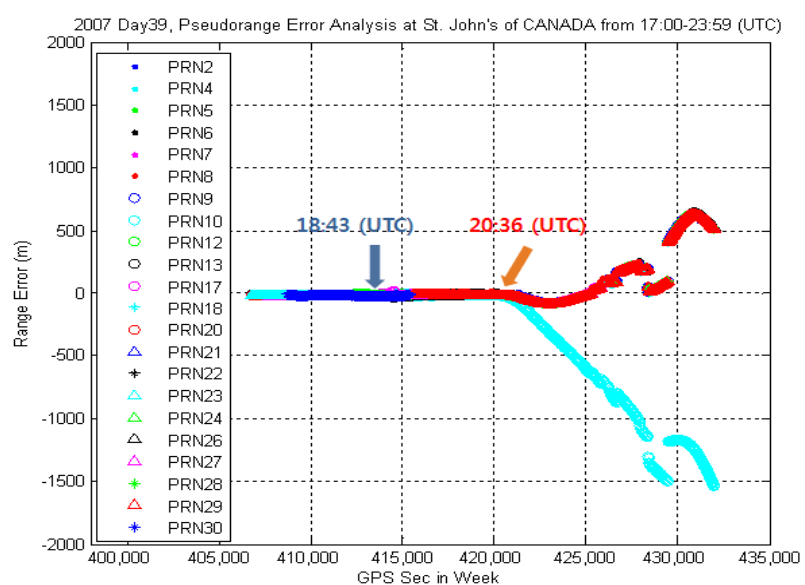

Fig. 5 Pseudorange error on 8th of Feb. (day 39) in 2007 from 17:00 23:59 (UTC) at St. John's of Canada
Fig. 5 shows the pseudorange error subtracted $P R_{O b s}$ from $P R_{C a l}$. More than $1.5 \mathrm{~km}$ of pseudorange error occurred on day 39, and it also shows that the other satellites were affected by PRN10's anomaly. Unusable set time of PRN10 by NANU was from 18:43 (UTC) but the actual anomaly was started from 20:36 (UTC).

Fig. 6 is the satellite clock bias during GPS anomaly occurred from 17:00 23:59 (UTC). GPS satellite has very accurate clock such as an atomic clock so it is very stable as shown in Fig. 6.

The receiver clock bias is a dominant factor to affect on pseudorange. Therefore, the result of receiver bias during GPS anomaly is shown in Fig. 7. It is known that the receiver clock bias is similar with Fig. 5 results except PRN10.

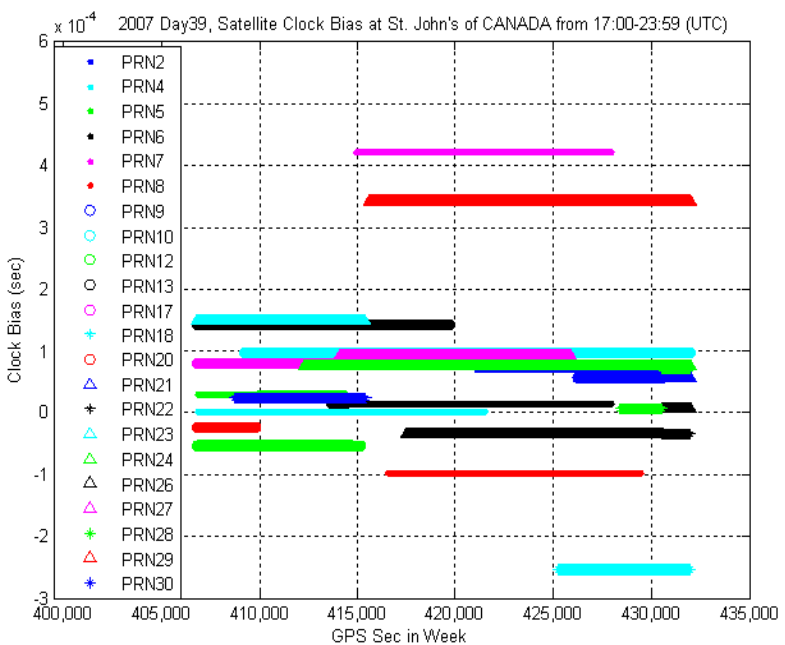

Fig. 6 Satellite clock bias during GPS anomaly from 17:00 23:59 (UTC)

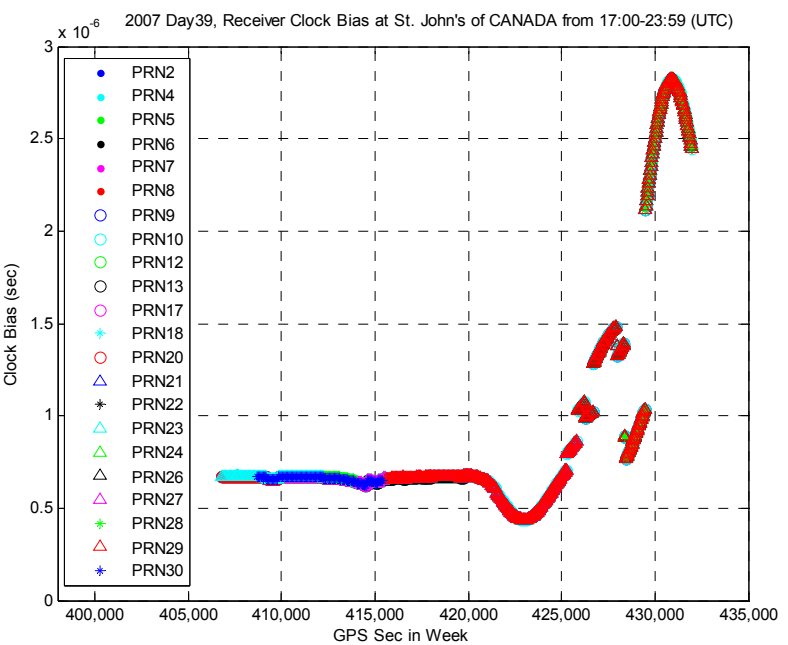

Fig. 7 Receiver clock bias during GPS anomaly from 17:00 23:59 (UTC) 


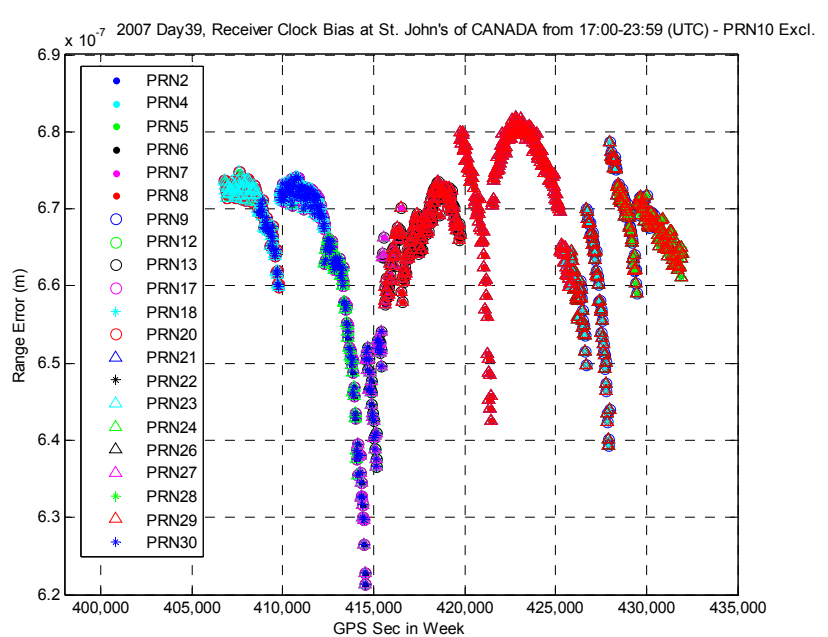

Fig. 8 Receiver clock bias eliminated anomaly satellite (PRN10) from 17:00 23:59 (UTC)

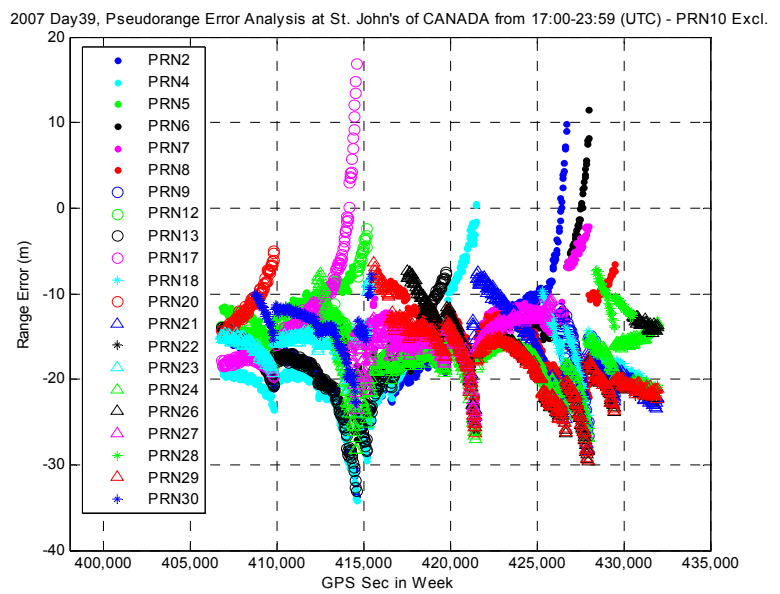

Fig. 9 Pseudorange range error eliminated anomaly satellite (PRN10) from 17:00 23:59 (UTC)

\subsection{Corrected Results}

The receiver clock bias results eliminated PRN10 are shown in Fig. 8 and the clock bias is decreased compared to Fig. 7 results by eliminating PRN10. It is considered that the discontinuity of the receiver clock bias in Fig. 8 is related to the change of visible satellites' number.

PRN10 occurred anomaly was excluded and the corrected pseudorange results are shown in Fig. 9. Compared to Fig. 5 results, the pseudorange errior is decreased within $50 \mathrm{~m}$ range error, and it is known that $\mathrm{PRN10}$ caused the pseudorange error and affected other satellites' pseudorange. The results also show that there is common bias around $15 \mathrm{~m}$, and it is considered as the measurement noise.

\section{Conclusions}

Increasing cases of GPS anomaly occur continuously according to the degradation of GPS performance, and there are many interests in the integrity monitoring of GPS signals all over the world. There are some main factors to cause GPS anomaly such as satellite clock failure, navigation message error, ephemeris error and equipment fault in the case occurred on GPS satellite. GPS anomaly can be resulted in pseudorange error to GPS users and also can cause a lot of economic loss sometimes. Then it is important to analyze the anomalous phenomenon of GPS signals and to devise the correction method to improve the pseudorange accuracy.

In this paper, the basic concept of how to detect the anomaly case was presented and the correction method to improve the pseudorange accuracy was also proposed when the anomaly satellites were monitored.

The paper showed the representative anomaly cases classified as for main fault types, and SVN40/PRN10 anomaly case was analyzed occurred an ephemeris error. The pseudorange error of PRN10 increased more than $1.5 \mathrm{~km}$ and the other satellites were also affected on pseudorange error due to the ephemeris error occurred on PRN10.

According to the proposed concept for improvement of pseudorange accuracy, PRN10 was eliminated and the corrected results were shown compared with the results of when the anomalous satellite was used. The results eliminated PRN10 showed enhanced accuracy in pseudorange error less than $50 \mathrm{~m}$ error range.

From the results, it is known that the pseudorange accuracy can be improved by eliminating the error-occurred satellite when it is monitored that there is anomalous signal. Also, the real-time integrity monitoring should be considered for increasing IM performance as a future work.

\section{Acknowledgments}

This research was supported by a grant (06-A03, PMS1920) from R\&D Program Funded by Ministry of Land, Transport and Maritime Affairs of Korean government.

\section{References}

[1] AISM-IALA (Association Internationale de Signalisation Maritime - International Association of Marine Aids to Navigation and Lighthouse Authorities) (2004), "IALA Recommendation R-121 On the Performance and Monitoring of DGNSS Services in the Frequency Band 283.5-325 kHz Edition 1.1", Appendix 1.

[2] B. Hofmann-Wellenhof, H. Lichtenegger and J. Collins (2001), "Global Positioning System: Theory and Practice", 
Springer published.

[3] Cho, D., Park, S., Choi J. and Suh, S. (2007), "A Study on Integrity Monitoring Improvement of the DGPS Reference Stastion", Journal of Korean Navigation and Port Research, vol.31, no.6, pp.509-514.

[4] Enge, P., Phelts, E. and Mitelman, A. (1999), "Detecting Anomalous Signals from GPS Satellites", Draft Stanford University Report.

[5] GPS Advisories/NANUs, United States Coast Guard Avail at: ww.navcen.uscg.gov, [Accessed 22 June 2010].

[6] Gao, G. X., Tang, H., Blanch, J. and Lee, J. (2009), "Methodology and Case Studies of Signal-in-Space Error Calculation: Top-down Meets Bottom-up", Proceedings of the Institute of Navigation, September 22-25, pp.2824-2831.

[7] Korea Hydrographic and Oceangraphic Administration (2005), "A Study on the Method of GPS Signal Monitoring, Fault Detection and Identification, and Broadcasting", Research andDevelopmentof Korea WADGPS, SIS analysis, and Time synchronization of NDGPS station (III), September.

[8] Phelts, R.E., Akos, D.M. and Enge, P. (2000), "Robust Signal Quality Monitoring and Detection of Evil Waveforms", Proceedings of the Institute of Navigation 2000, pp.1180-1190.

Received 1 July 2010

Revised 5 September 2010

Accepted 9 September 2010 